\title{
A Kind of Cross-layer Routing Algorithm considering MAC Collision (CLRA-MC) in Multi-hop Ad-hoc Networks
}

\author{
Xu Li, Si Li, Ying Liu, Chenchen Ma and Haoxiong He \\ School of Electronics and Information Engineering, State Key Lab. of Rail \\ Traffic Control and Safety \\ Beijing Jiaotong University, Beijing, China \\ xli@bjtu.edu.cn
}

\begin{abstract}
Multi-hop Ad-hoc networks is an important organization way for Internet of Things. Through the simulation and analysis in high data load conditions, it can be seen that packet loss is not mainly caused by the change of data links, but the data collisions in MAC layer. What's more, the data collisions affect the routing constructions in NET layer. Therefore, packet loss is not only related to the routing algorithm, but also closely connected with point-to-point transmission performance in MAC layer. So we proposed a cross-layer scheme between the MAC layer and the NET layer. Through the simulation and analysis, it can be proved that this scheme improves the packet delivery, decreases the end-to-end delay and reduces the routing cost, applies to low speed mobile net-works and high data load scene.
\end{abstract}

Keywords: Multi-Hop Ad-Hoc Networks, Packet Loss, Data Collision, Cross-Layer Routing Algorithm

\section{Introduction}

In Internet of Things, the network has more terminals and higher data load than the current network. Multi-hop ad-hoc network is an important organization way of Internet of Things. Although much progress has been made in the ad-hoc network, the routing is still a very challenging problem. Most researches focus on the routing problems which are caused by the changing topology and networks congestion [1-4] now, but less regarding about the collisions and data load in MAC layer. It can be seen that packet loss is mainly caused by the data collision in MAC layer, not the change of links in high data load scene through the simulation and analysis in this paper. So a new cross-layer scheme is proposed to solve this problem. The scheme considers the information from MAC layer to choose routing, and it proves to be effective in improving network performance through the simulation.

The rest of the paper is as follows: in section 2, this paper reveals the current problems through the simulation and analysis; in section 3, the related research is introduced; the cross-layer scheme (CLRA-MC) between the MAC layer and the NET layer is proposed and analyzed through simulation in section 4 to 6 ; in section 7 , we conclude this paper.

\section{Discovery and Analysis of the Problems}

We use NS2 to simulate in different scenes and data load, track the TRACE files, and analyze the reasons of packet loss. There are mainly three types of packet loss:

(1) " RTR : CBK, MAC : RET" means: If handshake is still not successful after a node repeatedly sends RTS in the MAC layer, packets will be discarded. In this case, the node will test whether these packets meet the repairmen condition or not. If the condition allows, the uplink node will broadcast RREQ to repair routing. Conversely, the NET layer 
will discard the packets. It can be caused by the topology change, the collision in MAC layer and the bad conditions of transmission links.

(2) "IFQ : ARP” means: Packet loss is caused by the ARP in IFQ layer.

(3) “ RTR : NRTE” means: There is not available routing for the arriving packets. The reasons may be that the source node has begun to establish the routing but failed; or the packets have arrived in an intermediary

Node, but this node does not have routing to the destination node.

Then three scenes are set up as follows (the following simulation is based on the 802.11 MAC and AODV protocols):

(1) Dynamic scene and smaller data load (as shown in Table 1)

(2) Dynamic scene and larger data load (as shown in Table 2)

(3) Static scene and larger data load

Compared to scene (2), the third scene is static. The mobile velocity is $0 \mathrm{~m} / \mathrm{s}$. Other parameters are the same.

Table 1. Simulation Environment 1

\begin{tabular}{|c|c|}
\hline Scene Settings & $1000 \times 1000 \mathrm{~m} 2$ \\
\hline Node Num & 40 \\
\hline Pause Time of Nodes & $2 \mathrm{~s}$ \\
\hline Simulation Time & $100 \mathrm{~s}$ \\
\hline Mobile Velocity & {$[4,6] \mathrm{m} / \mathrm{s}$} \\
\hline Data Type & $\mathrm{cbr}$ \\
\hline Max Connections & 10 \\
\hline Packet Size & 512 bytes \\
\hline Packet Rate & 1 packet/s \\
\hline
\end{tabular}

Table 2. Simulation Environment 2

\begin{tabular}{|c|c|}
\hline Scene Settings & $1000 \times 1000 \mathrm{~m} 2$ \\
\hline Node Num & 40 \\
\hline Pause Time of Nodes & $2 \mathrm{~s}$ \\
\hline Simulation Time & $100 \mathrm{~s}$ \\
\hline Mobile Velocity & $\mathrm{cbr} / \mathrm{m}$ \\
\hline Data Type & 32 \\
\hline Max Connections & $\begin{array}{c}1000 \text { bytes (the maximum } \\
\text { without splitting data } \\
\text { packet })\end{array}$ \\
\hline Packet Size & 8 packet/s \\
\hline Packet Rate & \\
\hline
\end{tabular}

After analyzing the TRACE files, some problems have been found. In the first scene, the source nodes totally send 745 packets, and the destination nodes receive 715 packets, the delivery rate is $95.97 \%$. In this simulation, 30 packets are lost, and $86.67 \%$ are the type of "RTR : CBK, MAC : RET". In the second scene, when the 
data load increases, this loss type also increases. In the third scene, packet loss caused by "RTR: CBK, MAC: RET" is more, and most loss is caused by the unsuccessful RTS/CTS handshakes. It can be seen that the same result happens in the first and second scenes.

Through the simulation and analysis, especially in high data load scenes, it can be found that packet loss is aroused by a large number of data collisions in MAC layer and data link's instability in NET layer. So the packet loss is not only connected with the routing algorithm itself, but also related to the point-to-point transmission performance in MAC layer.

\section{Related Research}

Paper [5] proposes a Delayed on-Demand QoS Routing (AODV-D) algorithm based on time delay. It considers the competitive information in the MAC layer to estimate the delay of competition, and the length of the nodes in interface queue to estimate queue delay. But the collision problem which is brought by the number of neighbor node or service load is not considered.

CEDAR routing [6] is suitable for extracting core of distributed Ad-hoc networks. It consists of three key components: establish and maintain the core nodes; use the transmission of enhanced wave and weakened wave to realize the spread of high bandwidth and reliable link state in the core nodes; the core nodes use local information. Bandwidth is the most sensitive QoS parameter in ad-hoc networks. So in this paper, the MAC layer and the NET layer closely cooperate to estimates available bandwidth. But the load factor of core nodes is not mentioned.

Paper [7] proposes a cross-layer scheme. It considers minimum hops in NET layer, link quality and congestion in MAC layer, wireless channel condition in PHY layer. In a scene which has fixed parameters, they analyze the performance of this scheme. But they don't verify the adaptability for different scenes.

In a word, there are fewer discussions about the influence of data collision in MAC layer and the instability of data link state on the routing establishment in high data load environment. Considering it, a cross-layer routing algorithm is proposed in this paper. In the condition of hardly sacrificing the system throughput, the scheme improves the packets delivery, decreases the end-to-end delay and reduces the routing cost in the largescale and mobile scenes.

\section{Cross-Layer Routing Algorithm (CLRA-MC)}

In the NET layer, routing selection is related to hops, link stability and so on. The delivery and scheduling strategy in MAC layer will influence the performance of packets delivery ratio and delay, etc. The performance of NET layer routing protocol is closely related to the quality of MAC layer. Therefore, Cross-layer Routing strategy mainly proposes two methods in this paper: one method is using RTS for maintaining neighbors in the MAC layer, and cancelling HELLO message for neighbor's maintenance in the NET layer at the same time. It is comparatively slow for the NET layer to judge the failure of links, and the information of overdue neighbors can live for the time ALLOWED HELLO LOSS * HELLO INTERVAL at most. Compared with HELLO message sent to the NET layer one second a time, the retransmission time of RTS in the Link layer is much shorter. Previous simulation results prove that detecting link state in the Link layer is better than testing connection information in the NET layer. Although the messages between NET and MAC layer are added, the consumption of controlling resources decreases and performance is promoted. The second method combines point-to-point link state in the MAC layer with the routing protocols to make optimized routing choice and improve the routing performance. So, based on the idea of cross-layer, this paper 
proposes a cross-layer routing optimization mechanism considering MAC collision (CLRA-MC).

The following routing metrics are expressed as follows in this paper:

(1)The MAC layer uses average data FTE (Frame Transmission Efficiency) to correctly reflect the link quality and congestion of each hop, and to provide basic information for the routing decision.

For example, node A and node B transmit data in point-to-point way, the MAC layer counts the number of packets sent by the MAC layer of node $\mathrm{A}$ in a cycle of $\mathrm{T}$ (as $\mathrm{N}$ ) and ACK received by node $A$ from node $B$ (as $n$ ).Then in the cycle of $T$, the probability of node A and node B's successful transmission is:

$$
F T E_{A B}=\frac{n}{N}
$$

For the multiple hops path between Node $\mathrm{S}$ and node $\mathrm{D}$, the value of $F T E_{S D}$ is got by multiplying FTE of every hop in the path, namely:

$$
F T E_{S D}=F T E_{S A} \times F T E_{A B} \times \ldots \times F T E_{M D}
$$

(2)According to the traditional routing algorithms, the minimal hop criterion HopMetric ${ }_{S D}$ can be the following formula:

$$
\text { HopMetric }_{s D}=\frac{\text { NodeNum }- \text { Hop Count }}{s D}
$$

In the formula, NodeNum represents the node number in the networks, HopMetric ${ }_{S D}$ is the hop counts of the path between node $\mathrm{S}$ and node D. Thus, in the process of choosing routing, the more hops the path contains, the smaller the value of HopMetric HD $_{S}$ is.

(3)Comprehensively considering the two above factors, we get the routing metric as follows:

$$
\text { RouteM etric }_{S D}=\text { HopMetric }_{S D} \times F T E_{S D}
$$

It aims at choosing a routing with better performance. If links' quality is better and hop counts are smaller, then the value of (4) is greater. That means the performance of routing is better.

\section{The Implementation of CLRA-MC}

The route metric field is added to the RREQ and RREP as routing metrics in AODV. The idea of renewing routing abides by the priority of serial number; the maximum serial number is the newest routing. If serial numbers are equal, the larger route metric is the optimal choice. At the same time, route metric field is added to the routing table of nodes as an attribute value of the routing.

Route metric field is initialized to zero. In the process of broadcasting and transmitting RREQ, a node gets the number of data packets and ACK which local node has received from one-hop nodes in a period of T. Then it calculates FTE value of the local node and one-hop nodes, calculates the hop metric value according to the RREQ's hop field, and the value of route metric is further calculated. The calculation method of route metric value is as follows: If the route metric field in the received RREQ is zero, route metric is equal to fte * hop metric; otherwise, route metric is equal to fte * hop metric * (rq -> route metric). A node calculates the values of route metric hop by hop, and adds them to the corresponding fields. When intermediate nodes receive routing request packets, they'll check this field to determine the process of routing-update. 
Similarly, in the forwarding process of RREP's unicast, node also needs link quality record in the MAC layer, calculates route metric value hop by hop, and adds them to the corresponding fields. When the intermediate node receives routing response packets, it'll check this field to determine the process of routing update. If the intermediate node receives the routing request which has the newest routing to the destination node, it'll get the value of route metric in the routing table from local node to the destination node as the initial value of route metric field to reply RREP, and RouteMetric ${ }_{S M D}$ value from the source node to the destination node will be the product of two segments of route metric value. The process is seen as Figure 1, Figure 2 and Figure 3.

In addition, it'll be discussed in the following that routing algorithm still supports the intermediate node response. When an intermediate node gives response, the value of routing metric is expressed as:

$$
\begin{aligned}
& \text { RouteMetric }_{S M D}=\text { HopMetric } \text { SMD }_{S M} \times F T E_{S M D} \\
& =\left(\text { HopMetric }_{S M} \times F T E_{S M}\right) \times\left(\text { HopMetric }_{M D} \times F T E_{M D}\right) \\
& =\left(1-\frac{\text { Hop Count }_{S M}}{\text { NodeNum }}\right) \times\left(1-\frac{\text { HopCount }_{M D}}{1-\text { NodeNum }}\right) \times F T E_{S D}
\end{aligned}
$$

When destination node gives response, the value of routing metric is:

$$
\begin{aligned}
& \text { RouteMetric }_{S D} \\
& =\text { HopMetric }_{S D} \times F T E_{S D} \\
& =\left(1-\frac{\text { HopCount }_{S M}+\text { Hop Count }_{M D}}{\text { NodeNum }}\right) \times F T E_{S D}
\end{aligned}
$$

Therefore, the D-value of the above two metrics is:

$$
\begin{aligned}
& \Delta=\left[\left(1-\frac{\text { HopCount }_{S M}}{\text { NodeNUM }_{1}}\right) \times\left(1-\frac{\text { HopCount }_{M D}}{\text { NodeNum }}\right)-\right. \\
& \left.\left(1-\frac{\text { HopCount }_{S M}+\text { HopCount }_{M D}}{\text { NodeNum }}\right)\right] \times F T E_{S D} \\
& =\frac{\text { HopCount }_{S M} \times \text { HopCount }_{M D}}{\text { NodeNum }^{2}} \times F T E_{S D}
\end{aligned}
$$

This value of " $\triangle$ "is very small, usually less than 0.01 (generally, number of nodes is less than 40 and routing hops count is in 6 hops), so it can be neglected. In the mechanism of cross-layer routing optimization, it can equally treat both the destination node and intermediate node's reply for the routing request. The reply of the destination node and intermediate node for the routing request can be equally treated.

Next, we consider the hops factor. Its value is expressed as follows:

$$
\text { HopMetric }_{S D}=\frac{\text { NodeNum }- \text { HopCount }_{S D}}{\text { NodeNum }}
$$

This value is calculated hop by hop like the FTE value, it is calculated hop by hop like the FTE value. The error analysis is as follows:

$$
\begin{gathered}
\square=\left(1-\frac{h_{1}}{N}\right) \times \ldots \ldots \times\left(1-\frac{h_{n}}{N}\right)-\left(1-\frac{h_{1}+\ldots \ldots+h_{n}}{N}\right) \\
=\frac{1}{N^{2}} \times\left[\left(h_{1} h_{2}+\ldots \ldots+h_{1} h_{n}\right)+\left(h_{2} h_{3}+h_{2} h_{n}\right)+\ldots . .+h_{n-1} h_{n}\right]
\end{gathered}
$$




$$
\begin{aligned}
& -\frac{1}{N^{3}} \times\left[\left(h_{1} h_{2} h_{3}+\ldots \ldots+h_{1} h_{2} h_{n}\right)+\left(h_{1} h_{3} h_{4}+\ldots \ldots+h_{1} h_{3} h_{n}\right)\right. \\
& \left.+\ldots \ldots+h_{n-2} h_{n-1} h_{n}\right] \\
& \quad+\ldots \ldots+\frac{1}{N^{n}} h_{1} h_{2} h_{3} \ldots \ldots h_{n-2} h_{n-1} h_{n} \\
& \quad+\ldots
\end{aligned}
$$

In the above format, the first bracket is the sum of $C_{n}^{2}$ product terms, and the second bracket is the sum of $C_{n}^{3}$ product terms, the nth brackets are the sum of $C_{n}^{n}$ product terms and so on. The value of " $\square$ " mainly depends on the value of the first term. And the more the hop counts are, the greater this value becomes. But the value of " $\square$ "is very small, so we can calculate hop by hop instead of by destination node. And if all of them are calculated by this method, the collision also can be reduced.

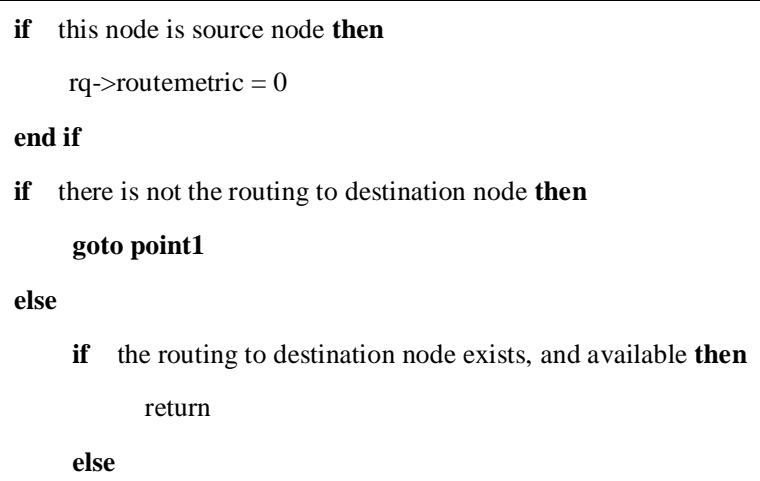

Figure 1. Algorithm of Sending Routing Request Packet (RREQ) 


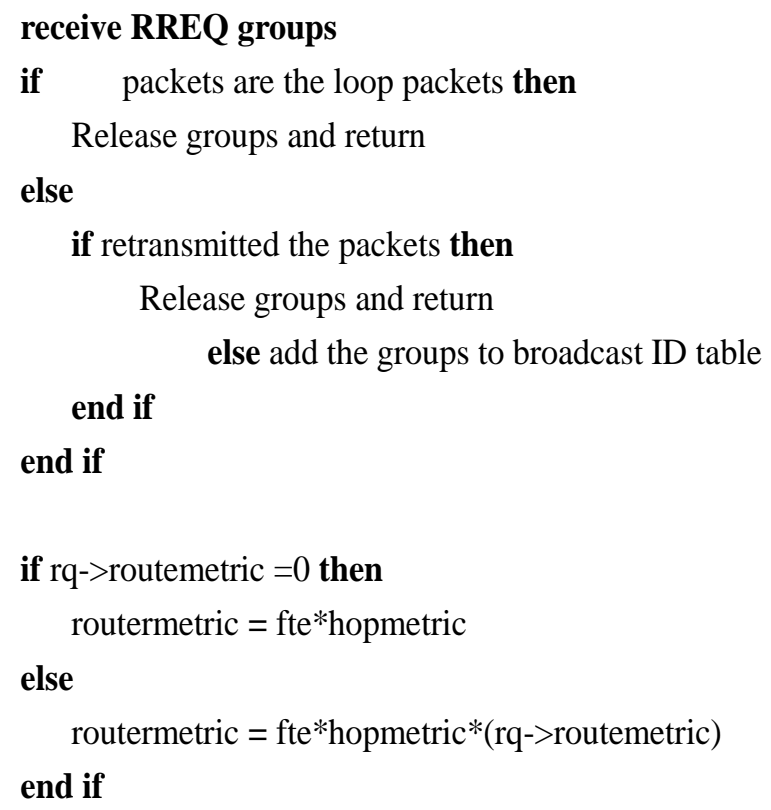

Figure 2. Algorithm of Receiving Routing Request Packet RREQ and Sending Routing Reply Packet (RREP)

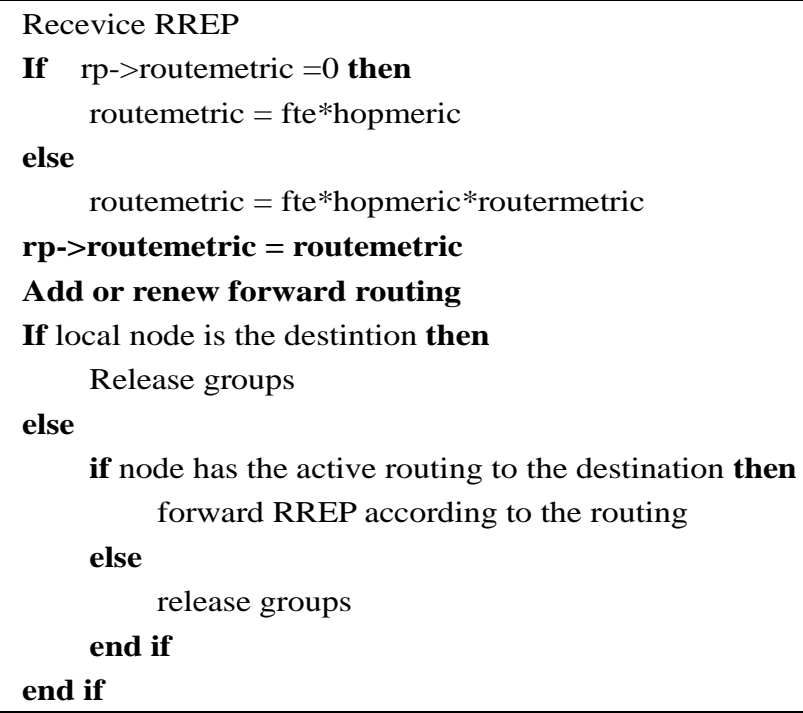

Figure 3. Algorithm of Receiving Routing Reply Packet (RREP) 


\section{Simulation and Analysis}

(1)Simulation conditions

The simulation conditions are shown in Table 3. Considering the change of the number of connections in the network, we respectively simulate and analyze the performance of CLRA-MC and typical AODV routing algorithm, including the comparison of delivery ratio, time delay and the overhead.

Table 3. Simulation Environment

\begin{tabular}{|c|c|}
\hline $\begin{array}{c}\text { Simulation } \\
\text { Software }\end{array}$ & NS2 \\
\hline Protocol & $802.11 \mathrm{MAC} /$ AODV \\
\hline Scene Settings & $1000 \times 1000 \mathrm{~m} 2$ \\
\hline Node Num & 50 \\
\hline $\begin{array}{c}\text { Pause Time of } \\
\text { Nodes }\end{array}$ & $2 \mathrm{~s}$ \\
\hline Simulation Time & $100 \mathrm{~s}$ \\
\hline $\begin{array}{c}\text { Max Mobile } \\
\text { Velocity }\end{array}$ & $1 \mathrm{~m} / \mathrm{s}$ \\
\hline Data Type & Cbr \\
\hline Max Connections & $5 、 10 、 20 、 25$ \\
\hline Packet Size & 512 bytes \\
\hline Packet Rate & 2 packets/s \\
\hline $\begin{array}{c}\text { Num of Random } \\
\text { Seed }\end{array}$ & 4 \\
\hline
\end{tabular}

(2)Analysis of simulation results

Regardless of what the network status is, CBR data always sends packets by a stable rate. So the packet delivery ratio can reflect the transmission efficiency of CBR service at most. Average end-to-end delay is one of the metrics to evaluate the service quality of the network. To the wireless ad-hoc networks with limited resources, overhead is also very important. Therefore, we compare the performance of the protocol in three aspects: packet delivery ratio, average end-to-end delay and the overhead of routing control. In the simulation, five different kinds of communication connections are used: 5, 10, 15, 20 and 25 which indicate the changes of networks load: increasing number of communication connections means that the network load is heavier and the number of the communication nodes is more.

For the three kinds of performance indicated above, we have separately written the corresponding AWK files to analyze the TRACE files which record the process of networks simulation, and use GNUPLOT for analyzing results (shown in Figure 4, Figure 5, Figure 6).

(1) Packet delivery ratio: the proportion of the packets number received by destination node and packets sent by the source node. It reflects the reliability of the networks transmission, and the higher the delivery rate is, the greater the reliability is (as the equation 10). From the Figure 4.1, it can be seen that CLRA-MC indeed improves the network's packet delivery ratio. When the number of communication connections is smaller, the two packet delivery ratios are close. That is because AODV's performance is good. When the number of communication connections is larger, the difference of two protocols in the performance is obvious. Although packet delivery ratios of the two protocol all decrease in this case, delivery rate with CLRA-MC is higher than AODV protocol. So the CLRA-MC improves the performance, because it considers the link 
quality in the process of routing establishment and choice. The simulation results prove that CLRA-MC improves the packet delivery ratio.

(2) The average end-to-end delay: The average time of sending packets from Application layer of source node to the Application layer of destination node, including each node's queuing delay and transmission delay from source node to destination node. It can be used to evaluate the rate of routing-lookup and the routing efficiency (as the equation 11).

As shown in Figure 5, with the increase of communication connections, both algorithms show an upward tendency, but CLRA-MC is less than AODV in delay. This is because the link of the routing establishment by CLRA-MC is better.

(3) Overhead: It is used to measure the efficiency of the protocol. Overhead reflects the efficiency of node power and the extent of network congestion. The protocol with large overhead easily leads to network congestion and delay (as the equation 12).

As shown in Figure 6, when the communication connections decrease, the routing overhead is lower; on the contrary, the overhead is larger. The cost of CLRA-MC is lower than AODV. Although the revised algorithm partly expands control packets, overhead still reduces. That's because node chooses the better link and decreases the cost of routing reconstruction.

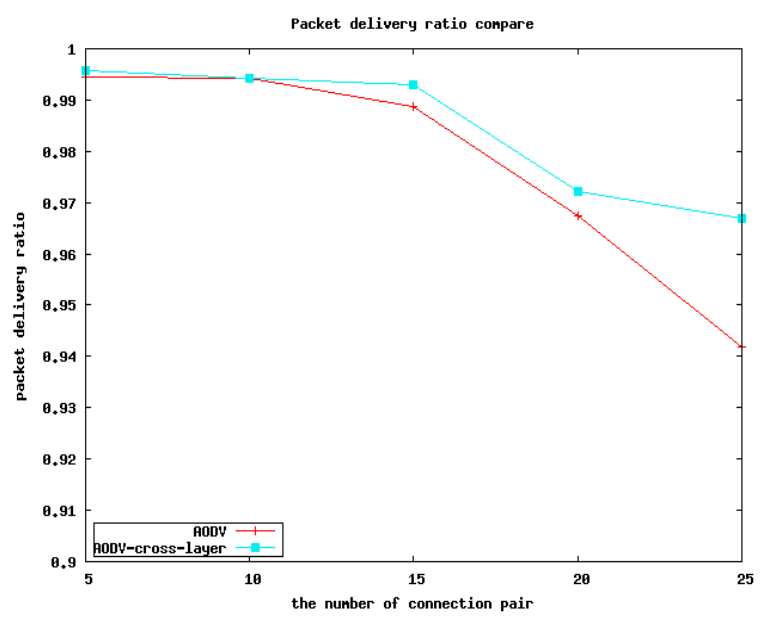

Figure 4. Comparison of Packet Delivery Ratio

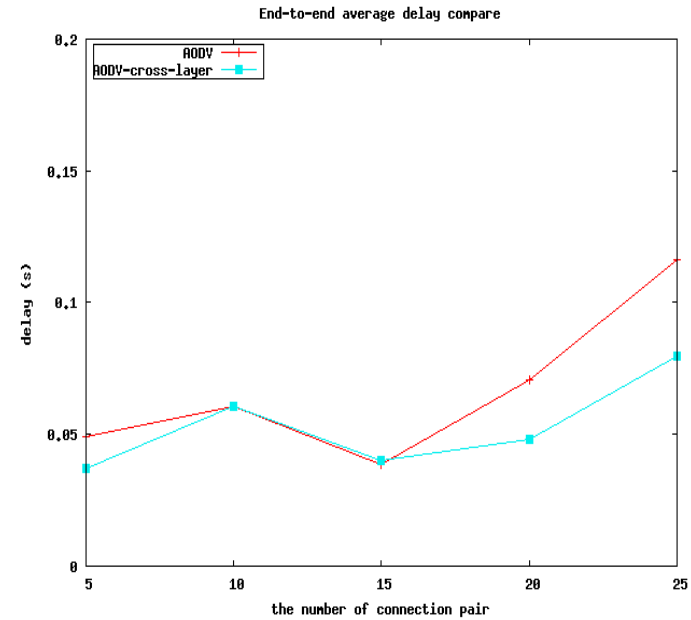

Figure 5. Comparison of Delay

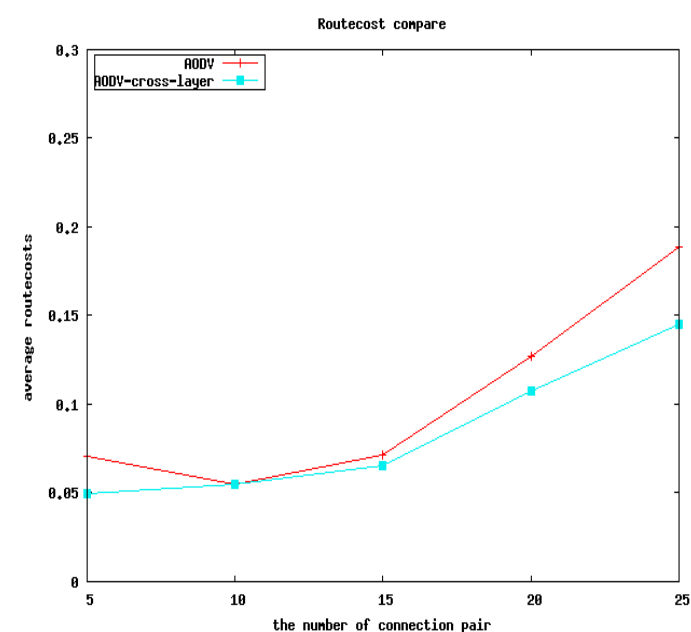

Figure 6. Comparsion of Cost 


\section{Conclusion}

Though analysis and simulation, we can conclude that the revised algorithm is suitable for low speed mobile networks and high data load scene. So the CLRA-MC can be applied to Internet of Things. In future, we will study different algorithms and their adaptability, which is the foundation of application. In addition, the algorithms research based on TDD will be the focus in our research group.

\section{Acknowledgment}

This work is supported by the NSFC (National Natural Science Foundation of China) No. 61371068 and No. 61172130, and supported by the MOST (Ministry of Science and Technology of the People's Republic of China) program No.2014BAK02B04. It is also granted by Fundamental Research Funds for the Central Universities No. 2014JBZ002.

\section{Reference}

[1] X. Xiaojing, W. Xin and Z. Zehua, "Self-Adaptive On-Demand Geographic Routing for Mobile Ad Hoc Networks", Mobile Computing, IEEE Transactions, (2012), pp. 1572-1586.

[2] P. R. Reshma and S. Bharathi, "An Adaptive Approach for Route Optimization in MANET", Information Communication and Embedded Systems (ICICES), (2013), pp. 706-712.

[3] W. Song, S. Jiang and G. Wei, "A Congestion-Aware Multipath Routing With Cross Layer Design for Wireless Mesh Networks", APCC, (2009), pp. 656-660.

[4] M. Naghshvar and T. Javidi, "Opportunistic Routing with Congestion Diversity in Wireless Multi-hop Networks”, INFOCOM, (2010), pp. 1-5.

[5] R. Kumar, M. Misra and A. K. Salje, "A Routing Protocol for Delay-Sensitive Applications in Mobile Ad Hoe Networks", International Symposium on Ad Hoc and Ubiquitous Computing, (2006), pp. 13-18.

[6] P. Sinha, R. Sivakumar, and V. Bharghavan, "CEDAR: A Core-extraction Distributed Ad Hoc Routing Algorithm”, IEEE JSAC, vol. 17, no. 8, August (1999), pp. 1454-1465.

[7] L. Jun, "A Cross-Layer Routing Optimization Method in Wireless Mesh Network", Software Engineering and Service Science (ICSESS), 2013 4th IEEE International Conference, (2013) May 23-25, pp. 357, 360.

[8] J. Y. Wu, W. J. Li, J. P. Huang, J. L. Zhang and D. R. Chen, "Key techniques for Mobile Internet:a survey”, SCIENTIA SINICA Informationis, vol. 45, no. 1, (2015), pp. 45-69.

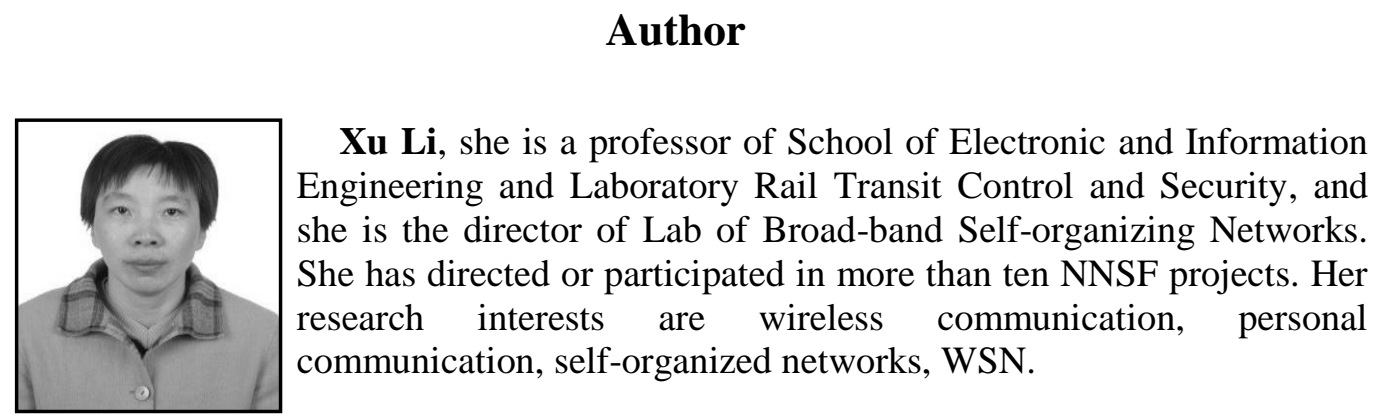

